oxygen. Our study indicates deficiencies in our practice in this specialist unit. There is a lack of clinical training and a low uptake of the self-assessment competency form. With these observations we are moving to make training and competency assessment mandatory for all clinical staff across the Trust. Also with the confusion we observed in correctly setting our hospitals traditional regulators we are considering switching to calibrated flow metres with a 'dial' to select flow which would reduce room for error and make oxygen administration safer.

\section{P92 TRAINING OPPORTUNITIES IN THORACIC ULTRASOUND FOR RESPIRATORY SPECIALIST TRAINEES IN THE WEST MIDLANDS DEANERY}

doi:10.1136/thx.2010.150979.43

T S Jordan, F Choudhary, R Heinink, S Mathew, I Hussain, M Allen. University Hospital of North Staffordshire NHS Trust, Stoke-on Trent, UK

Introduction The NPSA, ${ }^{1}$ supported by the BTS, ${ }^{2}$ recommends that chest drains for pleural effusions are placed using thoracic ultrasound (TUS). BTS pleural disease guidelines, currently under review, are likely to recommend the use of TUS. Royal College of Radiologists recommendations allow standardisation of TUS training, then assessment leading to Level-1 accreditation. ${ }^{3}$ The West Midlands Deanery, which comprises 13 Trusts providing 48 respiratory specialist training-posts, will require Level-1 competency in TUS as a CCT requirement. To achieve this there must be adequate training opportunities for trainees.

Methods and results We surveyed access to TUS training. Questionnaires were completed by 34 respiratory trainees from 11 Trusts (12 ST3, 9 ST4, 7 ST5, 1 ST6, 5 SpRs)-see Abstract P92 Table 1. Over half $(53 \%(18 / 34))$ were aware of Level-1 competency as a CCT requirement of which $33 \%(6 / 18)$ were unaware of criteria to achieve it. Most (91\% (31/34)) expressed concern about gaining sufficient TUS training for which responsibility was felt to be the Deanery's $(71 \%(24 / 34))$, the Trust $(26 \%(9 / 34))$ and the trainees's $(56 \%(19 / 34))$. Most had access to an US in their current Trust.

\section{Abstract P92 Table 1 Trainee responses}

\begin{tabular}{lr}
\hline & Number (\%) \\
\hline Number of TUS performed by trainees $(\mathrm{n}=34)$ & $16(47)$ \\
$1-20$ & $6(18)$ \\
$>20$ & $12(35)$ \\
None & \\
Training for those performing TUS ( $\mathrm{n}=22)$ & $11(50)$ \\
TUS course & $5(22)$ \\
TUS-competent supervisor only & $6(28)$ \\
Neither & \\
Not attended TUS course ( $\mathrm{n}=21)$ & $12(57)$ \\
Unable to get a place & $4(19)$ \\
Booked on course & $5(24)$ \\
Not tried & \\
Access to an US machine $(\mathrm{n}=34)$ & $11(32)$ \\
Yes-easily & $14(41)$ \\
Yes-with difficulty & $9(26)$ \\
No access & \\
\hline
\end{tabular}

Conclusion Much concern exists amongst respiratory trainees over TUS training and Level-1 accreditation. The majority (68\% (23/34) of trainees had not been on a TUS course mostly due to lack of availability, and were performing TUS without formal training; some of these without appropriate supervision. This clearly shows the need for greater access to recognised training opportunities in TUS. This may require local Trusts, in conjunction with the Deanery, to be more proactive in providing these opportunities and not rely on the availability of limited national courses.

\section{REFERENCES}

1. NPSA/2008/RRR003

2. British Thoracic Society Statement. BTS; Nov 2008.

3. Ultrasound Training Recommendations for Medical and Surgical Specialties. The Royal College of Radiologists 2005.

\section{P93 A SIMULATED BRONCHOSCOPY COURSE FOR NEW SPECIALIST TRAINEES}

doi:10.1136/thx.2010.150979.44

${ }^{1} \mathrm{~S}$ Mandal, ${ }^{2} \mathrm{~A}$ R C Patel, ${ }^{2} \mathrm{~J} \mathrm{~J}$ P Goldring. ${ }^{1}$ Department of Respiratory Medicine, Colchester University Hospital, Essex, UK; ${ }^{2}$ Academic Unit of Respiratory Medicine, UCL Medical School, London, UK

Introduction and objectives New trainees typically perform initial bronchoscopies on real patients under supervision. This model of training has safety and comfort implications relying on timely acquisition of skills, potentially compromised by a reduction in the number of bronchoscopies performed by trainees. Simulators have been validated in training novice practitioners. We present a model for initial bronchoscopy training by registrars for registrars.

Methods We developed a course based on survey data from NorthEast Thames trainees. We provided first year trainees with precourse material consisting of BTS guidelines and a presentation of bronchial anatomy. The day involved $1 \mathrm{~h}$ of theory, including indications, contra-indications, sedation, local anaesthesia, consent and management of complications. This was followed by a practical session with specialist nurses including assembling a bronchoscopy stack, troubleshooting and effective team-working. Most of the day was divided into three stations:

1. Use of two simulators (Accutouch, Immersion Corporation, USA), each trainee had $45 \mathrm{~min}$ of 1:1 tuition. Trainees were objectively scored using the validated Bronchoscopy Skills and Tasks Assessment Tool (Bronchoscopy International, USA). This score and freetext comments were sent to the trainees to discuss with consultants.

2. A slideshow of airway anatomy and pathology followed by MCQs addressing pre-course material.

3. Hands-on flexible bronchoscopy through a static bronchial tree model.

Feedback was assessed using a questionnaire at the end of the day Results $9 / 11$ trainees were within 3 months of starting StR training, 2/11 were within 12 months, having performed a median of five bronchoscopies (interquartile range 5-30). All trainees felt the course was pitched at the right level. They would all recommend this course to others, giving a rating of $4.8 / 5$. $90 \%$ thought the course should be carried out within the first 3 months of training. All participants would change their practice as a result of what had been learnt on the course. Suggestions for improvement included grey cases and even more hands-on simulation time.

Conclusions This potential model for bronchoscopy training was:

- Pitched at the right level.

- Delivered at an appropriate time.

- Received overwhelmingly positive feedback.

- Can be effectively delivered by experienced peers. 